\title{
Francesco Mastriani: Due Capitoli di Storia E di LetTeratura di NAPOLI E D'ITALIA
}

Francesco Guardian*

ABSTRACT: Si presentano qui due romanzi storici di Francesco Mastriani (1819-1891) che illustrano bene il punto di vista di molti intellettuali napoletani dopo l'unità d'Italia. In particolare: La figlia del croato (1867), descrive la risposta entusiastica per l'annessione del Veneto e di Venezia al nuovo Regno d'Italia a seguito della terza guerra d'indipendenza (1866); l'altro romanzo, Luigia Sanfelice / Due feste al mercato (1870 e 1876), chiarisce innanzi tutto il senso di delusione e fastidio causato dal 'romanzo napoletano' di Alexandre Dumas padre e quindi ne rettifica la prospettiva storica sugli eventi della Rivoluzione Partenopea del 1799. PAROLE CHIAVE: Francesco Mastriani; romanzo storico; Rivoluzione Partenopea.

RESUMO: Apresentam-se aqui dois romances históricos de Francesco Mastriani (1819-1891), que mostram claramente o ponto de vista de muitos intelectuais após a unificação da Itália. Em particular: o primeiro romance, La figlia del croato (1867), descreve a resposta entusiástica pela anexação do Vêneto e de Veneza ao Novo Reino de Itália em consequência da terceira guerra de indipendência (1866); o segundo, Luigia Sanfelice / Due feste al mercato (1870 e 1876), esclarece, principalmente, a sensação de decepção e de desagrado provocado pelo 'romance napolitano' de Alexandre Dumas pai e corrige a perspectiva histórica de Dumas

\footnotetext{
* University of Toronto - Canadá
} 
sobre os acontecimentos da Revolução Napolitana de 1799.

PALAVRAS-CHAVE: Francesco Mastriani; romance histórico; Revolução Napolitana.

ABSTRACT : Two historical novels by Francesco Mastriani (1819-1891) are presented here. They illustrate well the point of view of many Neapolitan intellectuals after the unification of Italy. The first novel, La figlia del croato (1867), describes the enthusiastic response to the annexation of Veneto and Venezia to the newly formed Regno d'Italia as a result of the third war of independence. The other novel, Luigia Sanfelice / Due feste al mercato (1870 e 1876), clarifies the sense of delusion and discomfort caused by the "Neapolitan novel" written by Alexandre Dumas father, and it rectifies his historical perspective on the Neapolitan Revolution of 1799. KEY-WORDS: Francesco Mastriani, historical novel, Neapolitan revolution. 


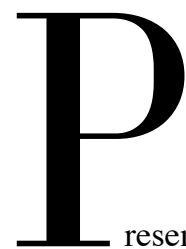

resento qui uno stralcio di un mio lavoro in corso su Francesco Mastriani (1819-1891).

Si tratta, in particolare, di due romanzi, ovvero di due episodi della ricchissima biografia intellettuale dello scrittore napoletano: La figlia del croato (1867) e Luigia Sanfelice / Due feste al mercato (1870 e 1876). ${ }^{1}$ Per la genesi de La figlia del croato si deve di necessità ricorrere a opere dello stesso Mastriani: a I vermi. Studi storici su le classi pericolose in Napoli (1863-64) ed a I lazzari. Romanzo storico (1865). La genesi di Luigia Sanfelice sta invece ne La San Felice (si noti la diversa grafia), romanzo fiume - di ben 1742 pagine nell'edizione consultata - di Alexandre Dumas padre, di cui Mastriani intende correggere la visione dei fatti di storia napoletana e italiana lì raccontati. ${ }^{2}$

1 Questo secondo romanzo, per le ragioni che si vedranno più sotto, si presenta con una doppia identità. Esce a stampa nel 1870 con il titolo Luigia Sanfelice e poi, con una brevissima nuova premessa e con il nuovo titolo, Due feste al mercato, nel 1876.

$2 \mathrm{Su}$ date di pubblicazione e vicende editoriali del romanzo di Dumas basti quanto si legge in apertura dell'edizione italiana Adelphi (vd. "Opere Citate") in cui, va osservato, il titolo originale, La San Felice, infelice invenzione di Dumas, è mutato nella grafia: "La Sanfelice apparve a puntate sul quotidiano parigino La Presse fra il 15 dicembre 1863 e il 3 marzo 1865 e, con uno scarto di qualche mese (10 maggio 1864- 28 ottobre 1865), sull'Indipendente, il giornale che proprio a Napoli Dumas aveva fondato e diretto. Pressoché contemporanea l'edizione in nove volumi di Michel 


\section{La figlia del croato (1867)}

La conclusione de I vermi, studi storici, riecheggia nella conclusione de I lazzari, romanzo storico, e consiste nella prima auspicata e poi descritta e pienamente realizzata emancipazione del popolano napoletano, non più lazzaro, luciano o candido-spontaneo senza identità individuale da mobilitare per fini reazionari. ${ }^{3}$ Già in questo puntare l'attenzione sull'uomo del popolo, sulla sua tradizione e sulla sua identità, per comprendere e spiegare i grandi eventi storici del passato prossimo, si delinea con chiarezza l'orientamento sociopolitico di Francesco Mastriani. In apertura de I lazzari, ovvero nelle pagine di dedica del romanzo al fratello Giuseppe, si dà per scontata, ovvero per già avvenuta, l'emancipazione del popolano. Che ora però deve essere istruito perché la partecipazione alla vita politica del paese richiede un continuo processo di informazione e di formazione. Con questo in mente, ben si intende come niente sia più utile alla formazione dei nuovi cittadini quanto la conoscenza storica. Il romanzo storico ha dunque per Mastriani, innanzitutto, una funzione educativa.

Visto l'immediato successo de I lazzari, questa funzione didattico-formativa del romanzo dev'essere sembrata ben avviata al suo autore. Centrato dunque l'obiettivo con il successo popolare, si apriva allo scrittore con più chiarezza la via maestra del romanzo storico. E tale sarà allora La figlia del croato di due anni dopo, ovvero del travagliatissimo anno de "La Domenica", settimanale scritto tutto di suo pugno, in cui il romanzo compare in appendice in prima edizione. ${ }^{4}$ Intanto, val la pena di notare che Mastriani aveva scritto e pubblicato I figli del lusso (1866), che sta a I vermi (1863-64) come La figlia del croato (1867) sta a I lazzari (1865): due séguiti di due romanzi di successo. ${ }^{5}$ Va aggiunto che il numero di lettori e la loro simpatia non corrispondevano certo alla serenità economica dello scrittore che, dopo essere stato licenziato dal Ministero dell'Interno il 17 aprile 1865 (cfr. Filippo Mastriani, Cenni 791) e dopo aver esaurito il denaro della liquidazione, si trovò in ristrettezze economiche disperate. ${ }^{6}$

Lévy, Parigi 1864-1865" (v. 1, p. 3).

3 Luciani sono i lazzari di Santa Lucia, fedelissimi alla corona borbonica. I candido-spontanei sono i lazzari che si opposero agli insorti del 15 maggio 1848; di loro dice Mastriani: "Vedemmo nel 1848 i Lazzari non più concordi nel sostenere le parti del dispotismo, ma bensì scindersi in due fazioni, di cui la più numerosa, la più compatta, la più intelligente tenere per i liberali ed avversare i candido-spontanei sguinzagliati dalla Camarilla di corte contro la costituzione." (p. 397).

4 "Nello stesso anno 1866, verso il mese di Novembre, [Francesco Mastriani] cominciò a pubblicare un giornale settimanale, col titolo La Domenica, di cui egli era l'unico scrittore. Egli scriveva l'articolo di fondo, il romanzo in appendice, la cronaca, le notizia teatrali, le biografie, i fatti varii e perfino le sciarade." (Filippo Mastriani, Cenni 794). 5 Per la verità, mentre I figli del lusso sono il dichiarato (dallo scrittore) seguito de I vermi, La figlia del croato segue I lazzari come romanzo storico.

6 "Quale tristissima, orrenda giornata fu per lo sventurato mio padre (e per noi tutti) quella di sabato 16 settembre 1865! Le irrimediabili angustie e privazioni del presente, un profondo scoraggiamento sull'avvenire della sua povera famiglia, accasciarono talmente lo sventurato, da farlo piangere sulla sterilità dell'ingegno e sull'egoismo degli uomini. La sua povera mente si aggirò tutto il giorno sui futuri consigli della disperazione. Queste parole non sono esagerate [...] ho letto, coi miei occhi, col cuore che mi sanguina dal dolore, in un libricino in cui egli segnava tutte le date memorabili della sua vita, queste terribili parole, colla data del 16 settembre 1865 . Privazioni, debiti, miseria, fame! Povero padre mio!" (Filippo Mastriani, Cenni, p. 793). 
Le cose non migliorarono l'anno dopo in cui, anzi, si ammalò di colera addirittura e si vide costretto a chiedere un sussidio pubblico all'allora sindaco di Napoli, barone Rodrigo Nolli. ${ }^{7}$

Dal novembre, sempre del '66, Mastriani reagì alla miseria diventando scrittore unico ed editore de "La Domenica", in cui pubblicò a puntate prima La brutta (dal 18 novembre 1866 al 6 gennaio 1867) e poi La figlia del croato (dal 6 gennaio al 1 settembre 1867). La genesi di questo secondo romanzo storico non dev'essere stata delle più tranquille, viste le travagliate circostanze di vita dello scrittore. Ma chiarissimi erano a questo punto per lui sia l'orientamento sociopolitico su cui imbastire la trama del romanzo, sia lo stile didattico-informativo con cui raggiungere il più ampio numero di lettori. C'era comunque una differenza notevole rispetto a $I$ lazzari e a tutte le altre opere precedenti che consisteva nella scelta dell'ambientazione italiana e non napoletana: un cambiamento di notevole importanza per lo scrittore nella percezione del proprio lavoro. Più che una mutazione di poetica, ovvero di cambio di prospettiva su natura, forma e ambito sociale della sua scrittura, si trattò di un esperimento. La figlia del croato costituisce infatti un episodio isolato rispetto agli altri lavori, precedenti e successivi. È facile tuttavia immaginare come nell'atto della scrittura Mastriani cominciasse a vedere la sua missione magistrale estesa a tutta l'Italia, ovvero alla nuova Italia che ormai includeva anche il Veneto e Venezia. Il cambiamento / esperimento non si riferisce allora soltanto al contenuto narrativo e all'ambientazione del romanzo, ma anche e soprattutto al pubblico stesso, ovvero ai lettori ideali immaginati e sperati per quest'opera: lettori di tutta l'Italia per un'opera sì storica, ma anche politica e attuale, informativa e giornalistica addirittura, vista la quasi contemporaneità dei tempi di scrittura e dei tempi degli eventi narrati. ${ }^{8}$

Argomento del romanzo, come si è sopra accennato, è la terza guerra di indipendenza - ma prima guerra del Regno d'Italia, cui fruttò il Veneto e Venezia - vista da una doppia prospettiva personale e patriottica: quella del narratore che, deus ex machina, interviene spesso con commenti, giudizi e suggerimenti al lettore, e quella dei due personaggi principali. Sono questi Gigia e Camillo, patrioti innamorati e contrastati, sudditi di sua maestà imperiale Francesco Giuseppe, anzi Kaiser Franz-Joseph come, sfoggiando anche in questo la sua conoscenza del tedesco, precisa lo scrittore-narratore. Sognano, lottano e infine vincono. Il loro è un trionfo amoroso e patriottico che apre loro una nuova vita nella nuova Italia.

Si è detto dei tempi di pubblicazione vicinissimi ai tempi dei fatti narrati. Questi ultimi hanno inizio all' "alba del 10 giugno del meraviglioso 1866", appena dieci giorni prima della dichiarazione di guerra all'Austria di Vittorio Emanuele II. Il messaggio alla nazione, in cui

7 Il finora ignoto episodio è certificato da un foglio autografo che ho reperito in antiquariato e di cui do conto in un saggio di prossima pubblicazione. Per la stessa ondata di colera, può essere utile ricordare, Luisa Sipari lasciò Napoli per Pescasseroli, dove partorì Benedetto Croce (1866-1952).

8 Mastriani comincia a scrivere il romanzo nel gennaio del '67. Il 3 ottobre '66 con il Trattato di Vienna, l'Austria aveva ceduto Venezia alla Francia che la passò all’ Italia dopo il plebiscito del 21-22 ottobre. 
egli si dichiara "prima soldato della indipendenza italiana", è appunto "dato in Firenze il 20 giugno 1866" e riportato per intero nel romanzo (cfr. p. 112-115). I dieci giorni intercorsi fra le due date sono appena sufficienti a condensare il viaggio di rientro a Venezia della "figlia del croato", Gigia Ransom, e l'innamoramento con il patriota padovano Paolo Camilli. La terza guerra d'indipendenza si attesta nel romanzo con l'arruolamento del Camilli fra i garibaldini, riuniti e addestrati a Bari e Barletta, mentre Garibaldi lascia Caprera per incontrarli e condurli alla vittoria ai piedi delle Alpi.

Si perdono a questo punto le tracce della Gigia che prepara comunque un clamoroso rientro in scena. Di Lizza e Custoza si parla fuori dalla narrazione nel corso di una delle lunghissime digressioni del romanzo. Nella conclusione troviamo "le trattative di pace che seguirono alla breve e disastrosa guerra, e la cessione di Venezia alla sua legittima madre, l'Italia [...] La storia di questi fatti è notissima ai nostri lettori” (p. 148).

In questo romanzo Mastriani sembra essere fortemente suggestionato dal Tasso della Gerusalemme Liberata, più volte ricordato nel corso della narrazione, e in particolare dalla figura di Clorinda. Sono della figlia di San Giorgio, infatti, le parole che vengono spontanee al "volontario Padovano" (p. 19), ovvero all'innamorato Paolo Camilli, nell'accomiatarsi dai compagni cospiratori di Venezia per unirsi ai garibaldini che si vanno raccogliendo in Puglia:

Ma s'egli avverrà pur che mia ventura

Nel mio ritorno mi richiuda il passo,

Della vecchia mia madre a te la cura,

E del fratello a me si caro io lasso.

Fallo, per Dio, Signor, che di pietate

Ben è degno quel sesso e quella etate.

Paolo che amantissimo era del Tasso, la cui lettura aveva temperato il suo spirito a nobili e grandi cose, si trovò su le labbra i versi mentovati, a cui avea fatto pochi leggieri mutamenti. (p. 20) ${ }^{9}$

Un'altra curiosità forse ascrivibile a un ricordo della Gerusalemme Liberata riguarda la particolare identità somatica di Gigia Ransom. Che è donna bruna pur avendo entrambi i genitori

9 Ecco l'ottava originale, tutt'intera, del Tasso:

Ma s'egli avverrà pur che mia ventura

Nel mio ritorno mi richiuda il passo,

D'uomo che'n amor m'è padre, a te la cura,

E de le mie donzelle io lasso.

Tu nel'Egitto rimandar procura

Le donne sconsolate e'l vecchio lasso.

Fallo, per Dio, Signor; che di pietate

Ben è degno quel sesso e quella etate. (GL XII.6) 
biondi di pura razza "imperiale", austriaca la madre e croato il padre. "Croato" è quanto dire forte e severo soldato dell'esercito austriaco. ${ }^{10}$ Ora bisogna fermarsi un attimo a riflettere di fronte a questa anomalia. È possibile, all'altezza del 1867, che Mastriani non avesse mai sentito parlare di Gregor Johann Mendel, e infatti qui fa un errato riferimento a una delle più elementari e ovvie leggi che regolano l'ereditarietà dei caratteri. Da coniugi biondi, infatti, non può che nascere prole dai capelli biondi. Il caso, cioè l'errore sul fronte della plausibilità, non sarebbe di per sè eccezionale se Mastriani, che ha un grande interesse per la scienza in generale, non avesse già dimostrato, nella Angiolina per esempio, un' attenzione particolarissima per l'eredità dei caratteri, non solo somatici, ma anche e soprattutto psicologici. Il carattere aristocratico di Angiolina, che tanto condiziona la trama di quel romanzo, riapparirà regale addirittura (come tipico di una immensa e ignara famiglia napoletana segretamente unita dallo stesso sangue) sia nella inflessibile Medea di Porta Medina che nel misterioso Figlio del diavolo.

Non è del tutto casuale, quindi, l'anomalia della Gigia dai capelli neri, veneziana italianissima, figlia di genitori biondi. L'anomalia è ostentata, anzi, e bisognerà pure spiegarla in qualche modo. Propongo di vedere in Gigia una rappresentazione simbolica proprio della Clorinda della Gerusalemme Liberata. La quale, si ricorderà, nacque bionda da genitori neri. Torquato Tasso, il cui verisimile faceva miracoli, l'aveva fatta figlia di San Giorgio di cui la madre di Clorinda era molto devota e di cui conservava un'icona prodigiosa nella sua stanzetta. Gigia Ransom, bruna figlia di genitori biondi, come Clorinda bionda figlia di genitori neri, sembra un'equazione interpretativa azzardata. Ma è ampiamente supportata dalla scena madre del romanzo, che ha luogo su un non specificato terreno di battaglia della terza guerra d'indipendenza. In un plotone di volontari garibaldini comandato dal sottotenente Paolo Camilli "volontario Padovano", si trovano Marietta Giuliani di Chiavenna, arruolata sotto il nome di Antonio Delfiore, ${ }^{11}$ e Lorenzo Venturi, un giovane veneziano di straordinari meriti militari che era divenuto il suo protettore:

Potea contare un diciassette anni: avea fatto le più disastrose e lunghe tappe senza mai dare segno alcuno di stanchezza; aveva chiesto al suo furiere una doppia razione di cartucce; si batté come un leone, e tirava come un vecchio granatiere. [...] avea due occhi scintillanti di ardor bellicoso: due gentili baffetti gli ornavano le labbra. (p. 130-131)

10 Questo significato non è certamente peculiare in Mastriani. "Croati” simili si incontrano anche nell'Ebreo di Verona di Bresciani, oltre che nel Sant'Ambrogio del Giusti.

11 La presenza di una donna nelle fila dei combattenti garibaldini è finzione narrativa che trova riscontro in noti episodi di patriottismo risorgimentale femminile, come quello di Antonia Masanello in Marinello che combatté con i Mille a fianco al marito Bortolo e quello di Colomba Antonetti Porzi che fu con Garibaldi sul Gianicolo nel ' 49 e perse la vita in battaglia. Sul fronte letterario la bella combattente in abiti maschili cronologicamente più vicina alla "figlia del croato" è il personaggio di Polissena nell'Ebreo di Verona di Antonio Bresciani. 
Questo giovane volontario veneziano, Lorenzo Venturi, prode, coraggioso, virile, protettore di donne... nè più nè meno valoroso della Clorinda tassiana 'protettrice' di Sofronia, è proprio lei, Gigia Ransom. Come Clorinda si rivela a Tancredi togliendo l'elmo e sciogliendo la chioma bionda, Gigia si rivela al padre ferito a morte e a Paolo Camilli, staccandosi i baffetti finti e togliendosi il berretto sotto cui si nascondeva una lunga treccia nera. Il croato benedice l'unione dei due innamorati prima di esalare l'ultimo respiro. "La breve e disastrosa guerra" (p. 148) ha fine. Venezia e il Veneto diventano parte del Regno d'Italia. Paolo Camilli ritorna a Padova "colla sua virtuosa e leggiadra sposa" (p. 148), raggiunge un'agiata posizione come agente di commercio, "e sarebbe felicissimo se l'ardente voto che egli forma ogni giorno, che tutti gl'Italiani sieno affratellati, indipendenti, liberi e temuti, avesse il desiderato compimento" (p. 148).

Caso unico nell'opera omnia di Mastriani, questo romanzo è fondato su un atteggiamento politico tutto italiano e non napoletano, come del resto s'è visto anche a proposito di ambientazione e personaggi principali. C'è solo un personaggio, minore, napoletano nel romanzo ed è tratteggiato in modo ironico e grottesco. Si tratta di "arciprete borbonico [...] faccia della sferica rotondità di certe parti del corpo, pancia della forma di un gran disco e tutto il resto in corrispondenza di queste membra sferoidali" (p. 68). L'arciprete, "schiuma del borbonismo" (p. 67), "sognava ad occhio aperto la così detta riciccillazione" (p. 69), ovvero il ritorno di Ciccillo / Franceschiello / Francesco II di Borbone.

C'è anche di napoletano nel romanzo una originale, patriottica interpretazione delle disastrose sconfitte di Lissa e Custoza. Si tratta di una lunga citazione che - è detto in una nota al testo Mastriani trasse da uno scritto di Giuseppe Piro del 1867: "Or bene domandiamo noi a nostra volta: fummo vinti in queste due giornate? A primo tratto pare che la risposta non possa essere dubbia, ma ponderandovi su, Custoza e Lissa, lo ripetiamo, furono due vittorie morali" (p. 117).

Molto resterebbe da dire per quel che riguarda la dimensione didattica del romanzo. Che si concentra soprattutto nelle lunghissime digressioni e nella menzione delle gloriose figure storiche italiane del Veneto e di Venezia. Le più vistosa digressione riguarda un intero capitolo, il settimo, sulla storia di Venezia dalla sua fondazione al tempo dell'occupazione austriaca, "senza tacere de' più grandi uomini che la resero illustre" (p. 89) da Marco Polo... ai Fratelli Bandiera.

Notevole è anche, sempre sul fronte didattico-formativo, la seria di riflessioni sociopolitiche della Gigia che, con ingenuità e ispirata saggezza, vede il mondo intero nel mezzo di una epocale trasformazione: non solo nella situazione veneziana e italiana, ma anche in quella generale del 
continente europeo e anzi dell'Europa e dell'America, la Gigia scorge segni di convergenza delle ragioni storiche e geografiche che portano alla libertà dei popoli oppressi. È la nozione stessa di impero - e di quello austro-ungarico in particolare - che per Gigia reca con sé un profondo senso di ingiustizia.

In fine, contribuiscono senz'altro ad alleggerire il tono di queste riflessioni e a cercare un'intesa con il lettore meno colto alcune ingenue espressioni di ironia antiaustriaca del narratore, che dice per esempio: "O aquila austriaca, tu hai due teste, ma sono teste di rapa" (p. 14); oppure afferma che la lingua tedesca a Venezia è un'assurdità, un "idioma che Carlo V dicea doversi parlare da' cavalli” (p. 27), per cui Gigia, che pur dovette parlare tedesco nell'infanzia, parla sempre in italiano perché si sente veneziana e italiana.

$$
* * *
$$

Se la forza d'urto de I vermi, romanzo veramente nuovo, si spendeva in varie direzioni, ovvero in vari aspetti della poetica dello scrittore - nella struttura, nella scelta dei temi, nel linguaggio, nell'organizzazione del materiale narrativo - è ovvio che essa si manifestasse anche nella trattazione dei temi storici. Che presero subito un orientamento sociologico e, nell'ineluttabile cammino "ascendentale" della umanità, si manifestarono soprattutto con l'emancipazione sociale del popolano, ovvero del ceto meno abbiente che a Napoli s'incarnava allora nella figura del lazzaro. A Napoli, si badi, non nel Regno della Due Sicilie, né tanto meno nel Regno d'Italia. Sicché quando personaggi e azione si trasferirono "in alta Italia", e cioè quando da I lazzari si passò a La figlia del croato, al nuovo romanzo storico venne a mancare il materiale umano originario, realistico, credibile e coinvolgente (anche per il lettore non napoletano) che assicurava la coerente linea di pensiero dell' autore, decisamente evolutiva se non proprio rivoluzionaria e patriottica. La figlia del croato è un romanzo più simbolico che realistico e, come abbiamo visto, i suoi personaggi sono obbedienti soldatini di piombo senza spessore psicologico. Con tutto ciò - siamo nel 1867, con un'Italia sempre più grande e più unita, con il re e il parlamento a Firenze, ma con Roma già capitale morale - il romanzo ebbe buona fortuna editoriale, come attestano le ristampe. ${ }^{12}$

Se consideriamo le difficili condizioni economiche, i disagi domestici, le condizione di salute, le incertezze del mercato editoriale... non possiamo non riconoscere qualcosa di prodigioso nella produttività di Mastriani in questo periodo. La figlia del croato (1867) "romanzo storico" è il

12 Si contano sei edizioni (o ristampe) dalla prima del 1867, in appendice de "La Domenica", all'ultima (Firenze: Salani) del 1935. Cfr. "Bibliografia dei romanzi” in Addesso et al., p. 297. 
seguito naturale de I lazzari (1865), così come I figli del lusso (1866) sono, in maniera ancora più palese, anzi dichiarata, il seguito de I vermi (1864) "studi storico-sociali". L'entusiasmo didattico-patriottico per temi storici sembra attenuarsi dopo La figlia del croato, mentre prendono sempre più rilievo, per l'autore, temi legati all'uguaglianza sociale e alla condizione della donna, anche se non mancano riferimenti e commenti di carattere prettamente storico nelle opere immediatamente successive. Dobbiamo comunque aspettare fino al 1870, anno della Breccia di Porta Pia, per un altro romanzo storico, o almeno così detto nel frontespizio.

L'anno stesso in cui completa I misteri di Napoli (1869-1870), Mastriani dà alla luce Luigia Sanfelice, un romanzo storico di complessa identità (se così si può dire) per il fatto che lo scrittore gli mutò il titolo qualche anno dopo, chiamandolo Due feste al mercato (1876). La storia di Luigia Sanfelice diventa la doppia storia di Luigia Sanfelice e di Eleonora Pimentel Fonseca, anzi prima questa che quella, se vogliamo dare la giusta cronologia alle due "feste", ovvero alle esecuzioni capitali delle due donne nella Piazza del Mercato nell'ambito della reazione borbonica alla rivoluzione del 1799 .

La storia è onnipresente nell'opera di Mastriani. Non a caso il novel, ovvero la forma narrativa del romanzo vero e proprio, che è poi prevalente fra le forme impiegate dallo scrittore, richiede ambientazione storica e caratterizzazione sociale della vicenda e dei personaggi. Si ritrovano elementi storici anche negli spunti riflessivi del narratore, sempre onnisciente, ${ }^{13}$ come nei commenti messi in bocca ai personaggi e nelle descrizioni di luoghi, ambienti e costumi.

Non sarà inutile, a questo punto, presentare una breve rassegna di note storiche colte nei romanzi che cronologicamente si pongono fra La figlia del croato (1867) e Luigia Sanfelice (1870). Già ne La brutta (1867) per la verità, dello stesso anno de La figlia del croato, ma apparsa prima ne "La Domenica", incontriamo un "infame sgherro della polizia borbonica" (3.32), Giuseppe Campagna, uno dei tanti "birri” irredimibili (con eccezione forse dell'ultimo, Luigi Aiossa, cui è dedicata Acaja) che popolano da personaggi storici le pagine del romanziere. Sempre ne La brutta troviamo anche "l'arresto del dottor Fulvi, imputato come mandatario di Mazzini per abbattere la monarchia napoletana e promuovere la repubblica, solita formula d'accusa con la quale si facevano i più arbitrarii arresti e le più illegali processure" $(3.39$, il corsivo è nel testo).

Più che a quella del romanzo storico, Le ombre (1867-68) è un'opera che si può ascrivere alla forma dello "studio storico-sociale", ideata con I vermi e consolidata con I figli del lusso.

13 La narrazione, in prima o in terza persona, in Mastriani è sempre al passato e appare frutto di una soluzione mnemonica variamente motivata e commentata. I ricordi sono, insomma, sempre filtrati dal narratore. Il quale poi, quando corrisponde allo scrittore Francesco Mastriani personaggio, diventa lui stesso una prova di veridicità della fabula, anche della più fantasiosa. Così è, per esempio, sia con Angiolina (1868), Memorie di una monaca (1879) e Karì-Tismé: memorie di una schiava (1882-83), in cui allo scrittore personaggio sono affidati dei memoriali che egli si impegna a pubblicare a stampa, e sia con Eufenia ovvero il segreto di due amanti (1868) e con Cenere o la sepolta viva (1877) in cui 'la storia' gli viene raccontata a voce. 
Questo vuol dire che troviamo ne Le ombre ben più presenti gli elementi tematici e retorici dell'anatomy che è forma diversissima dal romanzo vero e proprio. ${ }^{14}$ Oltre allo "storico" problema dell'inuguaglianza della donna, troviamo in questo romanzo elementi dell'ambientazione che appartengono alla storia nazionale, come questo, per esempio: “Il tempo era bellissimo; l'aere fresco e temperato. La brigata sen venne in su la strada di Porto or cantando l'inno di Garibaldi, or l' Addio, mia bella, addio, l'armata se ne va" (p. 453). ${ }^{15}$

In Una martire(1868), romanzo ambientato nel 1852, i riferimenti storici appaiono sulle pagine dei giornali: "Il commendatore [Ignazio Pasicci] era ancora a letto, e leggeva con molto diletto nel 'Giornale officiale' i particolari della caduta della repubblica in Francia” (p. 28).

Ne La contessa di Montes (1869) per rendere chiara l'ambientazione temporale si ricordano i fatti storici noti a tutti: "Era il tempo della guerra in Crimea [...] la visita fatta da Gladstone alle prigioni politiche napoletane e le sue rivelazioni al parlamento inglese" (p. 100).

Il figlio del diavolo (1869), in cui il diavolo mai nominato ma indicato da mille allusioni sarebbe re Ferdinando I, primo fornitore di 'orfani' alla Nunziata, è un romanzo di non facile classificazione. La componente storica è qui predominante su quella dell'invenzione narrativa; ma ai riferimenti a fatti storici accertati - dal trasferimento della corte borbonica a Palermo nel 1806 (I.36) all'arrivo di Gioacchino Murat a Napoli nel 1808 (I.31), dai briganti presi nella Storia del Reame di Napoli del Colletta (42-43) alla corrispondenza epistolare fra la regina Carolina e il brigante Mammone (II.2) - si aggiungono aneddoti di personaggi di corte, di briganti, di sanfedisti, di congiure e società segrete che sembrano più immaginari che reali.

Luigia Sanfelice (1870) ovvero Due feste al mercato (1876)

La genesi (come l'identità retorica) del romanzo appare condizionata dall'apparire a rimorchio de La San Felice di Alexandre Dumas padre. È certo che la lettura del romanzo francese, apparso a Napoli sull'“Indipendente" dal 10 maggio 1864 al 28 ottobre 1865, proprio a ridosso de I lazzari (Napoli: Gargiulo, 1865, in cinque volumi), deve aver suscitato una forte reazione negativa cui Dumas, ormai di nuovo a Parigi e fuori in ogni senso dall'esperienza napoletana, non si è curato di rispondere. Ma è indubbio che Mastriani, interpretando anche il desiderio di molti, se non tutti, i suoi lettori, abbia sentito il bisogno di reagire e di emendare in qualche modo la prospettiva storica e i giudizi di merito del francese. Il tono, comunque, non vuole essere polemico. Si apre, infatti, Luigia Sanfelice. Romanzo storico del Mastriani che già presenta nel titolo una correzione al romanzo francese (La San Felice), con questa programmatica dichiarazione:

14 Ricordiamo: "io non scrivo un romanzo" (I vermi); "chi vuole il fatto narrativo legga qualcos'altro" (I figli del lusso).

15 Realisticamente parlando questo alternarsi dei due canti risorgimentali non sembra troppo credibile o plausibile. E naturalmente quando si esce dal realismo si entra in un ambito più simbolicamente rappresentativo. Le due canzoni sono qui, allora, proprio per evidenziare l'identità risorgimentale della "brigata". 
Invitato a presentare, sotto la forma del romanzo storico, i fatti del nostro paese sotto il nome di una delle più illustri vittime della borbonica restaurazione in Napoli dopo le rivolture del 99, LUIGIA SANFELICE, stimai attenermi più alla dipintura degli attori principali di quel lugubre dramma; perocché già la Sanfelice rinata sotto la fervida e immaginosa penna d'uno dei più popolari romanzieri francesi, Alessandro Dumas padre. Nel trattare lo stesso subbietto, non mi allontanai dalla storia che in quel che poteva aggiugnere maggior colorito al tristo quadro di quella crudele reazione, di cui forse svolgerò, Dio permettendo, in altro lavoro, qualche altro importante episodio. Agosto, 1870. F.M.

La dichiarazione si può leggere come conferma delle reazioni negative suscitate dal libro di Dumas, e quindi come notizia di un avvenuto appello al Mastriani stesso per la scrittura di una rettifica, ovvero di un "romanzo storico" veritiero. L'autore è infatti "invitato a presentare [...] i fatti del nostro paese sotto il nome di [...] Luigia Sanfelice". E cioè gli è stato chiesto esplicitamente di dire il vero, di "presentare i fatti", di presentarli da un napoletano ("i fatti del nostro paese") e di presentarli facendo rifacimento al romanzo scritto da Dumas ("sotto il nome" della Sanfelice) "con fervida e immaginosa penna" e non certo con quella dello storico obiettivo e veritiero.

Mastriani si sente insomma gentilmente obbligato a scrivere sotto il nome della Sanfelice, ma è chiaro a lui come agli altri che il titolo del romanzo di Dumas (prima che del suo, ritoccato) non rispecchia affatto il contenuto dell'opera. Questo (presunto) ragionamento del Mastriani è certamente in linea con la decisione di ristampare anni dopo il romanzo con un altro titolo, Due feste al mercato (1876), senza il fastidio per il lettore di un obbligato confronto con Dumas. ${ }^{16}$

Sempre nella dichiarazione proemiale, Mastriani promette, dopo aver onorato l'obbligo derivato dall'accettazione dell' invito a scrivere i fatti del nostro paese sotto il nome di Sanfelice, di descrivere "qualche altro episodio" della reazione borbonica alla rivoluzione del 1799, cosa che aveva fatto già ne I vermi (1863-64) e nei freschi di stampa Misteri di Napoli (1869-70), e che farà anche nel Campanello dei Luizzi (1885) e nel Padrone della vetraia all'Arenaccia (1890). Quasi a giustificarsi del non cercato ma necessario scontro più storico-sociologico che letterario con il celebratissimo Dumas, Mastriani scriverà nell' "Avvertenza" preposta a Due feste al mercato (1876):

16 Il nuovo titolo, ricordo, si riferisce alle esecuzioni, in Piazza del Mercato di due protagoniste della rivoluzione e della Repubblica Partenopea del ‘99, Eleonora Pimental Fonseca e Luigia Sanfelice. 
Questo mio storico lavoro fu pubblicato in Napoli per la prima volta cinque anni or sono, con altro titolo che poco rispondeva al concetto dell'opera. Ebbi allora appena il tempo di correggere le prime pruove di stampa. Poca pubblicità si ebbe il mio libro, avvegnaché importante ne fosse il subbietto. Oggi con titolo più adatto ripubblico il mio lavoro.

Insomma il confronto con Dumas c'è stato, ma non ha generato grande interesse e il romanzo si può ripresentare tale e quale sei anni dopo con altro titolo e quindi come opera indipendente da qualsiasi condizionamento esterno. Fatto sta che l'esistenza stessa del romanzo di Mastriani non si spiega senza quella della San Felice di Dumas. Proviamo allora a mettere a fuoco alcuni elementi di quest'opera che sicuramente fecero inarcare le ciglia non soltanto a Mastriani, ma a tutti i lettori napoletani dell'“Indipendente" su cui essa apparve.

Dice Ena Marchi che "Dumas amava Napoli [...] l'amava di un amore appassionato, fervido - e non ricambiato: i napoletani non capirono "L'Indipendente" e diffidarono sempre del suo fondatore" ("A Napoli in nome del padre e della rivoluzione", in MARCHI, 1999, p. 1750). Vediamo ora qualche segno di questo grande amore e magari cerchiamo anche di capire come mai i napoletani non si sentirono tanto amati. Per cominciare, nella premessa al volume della San Felice - premessa 'riassuntiva' nel senso che venne scritta dopo la pubblicazione del romanzo a puntate - Dumas scrive:

La vicenda si svolge nel periodo del Direttorio compreso tra il 1798 e il 1800. I due eventi principali sono la conquista del regno di Napoli da parte di Championnet e la restaurazione di Ferdinando IV ad opera del cardinale Ruffo. (DUMAS, 1999, p. 15)

Della Sanfelice nemmeno l'ombra. Ma non soffermiamoci su questo, per ora. Si sa che un conto era l'intenzione iniziale dichiarata dal titolo e altro è quello che venne fuori alla fine, quasi un anno e mezzo dopo. ${ }^{17}$ Fatto sta che "i due eventi principali" sono presentati come straordinari, anzi inverosimili: innanzitutto, che Jean Étienne Championnet, abilissimo generale con meno di

17 La prima "appendice" del romanzo vede la luce su "La Presse" di Parigi il 15 dicembre 1863 e l'ultima vi appare il 3 marzo 1865. Sull'“Indipendente" il romanzo comincia a uscire nel maggio del 1864. 
un sesto delle truppe borboniche (10.000 vs 65.000), riuscisse a impadronirsi di Napoli, città di cinquecentomila abitanti con tre giorni d'assedio; e poi che il cardinale Fabrizio Ruffo, partito da Messina con cinque uomini, arrivasse a Napoli con quarantamila sanfedisti a rimettere sul trono re Ferdinando. Ed ecco il commento dello scrittore innamorato: "Solamente Napoli, con il suo popolo ignorante, volubile e superstizioso, poteva trasformare in eventi storici dei fatti così inverosimili" (DUMAS, 1999, p. 15).

A proposito dell' evento numero uno, ossia della "conquista [...] di Championnet", bisogna aggiungere che la rivoluzione napoletana del "99 è da Dumas chiamata "la rivoluzione di Championent", che è perfino paragonata a quella di Masaniello rispetto alla quale mostra "un'immensa differenza e soprattutto un immenso progresso" (p. 983). Championnet è presentato come un semidio, eccelso campione di alta cultura (più colto di un professore di archeologia romana), di saggia e benevole umanità per non parlare di cortesia che è chiaramente il suo forte. Poi ci sono le doti militari: Championnet è avveduto, previdente, prudente, deciso e audace. Su tutte primeggiano comunque le doti tipicamente francesi. Championnet è leale e sprezzante. Insomma la perfezione personificata. Gli sta alla pari soltanto l'ammiraglio Nelson, fra i mille personaggi del romanzo, alla cui memoria serba una profonda devozione:

Gli uomini come lui sono un prodotto della civiltà universale, che i posteri, senza far distinzione di nascita e di paese, considerano parte della grandezza del genere umano, il quale deve circondarli di riverente amore e ammirarli con immenso orgoglio. (p. 38)

Ecco, riesce proprio difficile immaginarselo l'orgoglio dei napoletani lettori dell' "Indipendente" per il guerriero figlio di Albione che gli ha impiccato Francesco Caracciolo. ${ }^{18}$ A Dumas piacciono gli uomini grandi e a Napoli, ahimé, non ne trova. Napoli "prima tappa dell'Oriente" (p. 281) è per lui un posto esotico e primitivo, un serraglio di orrori ancora non del tutto pronto a ricevere la civiltà che viene dalla Francia. Re Ferdinando per lui è un mostro di natura. Lo descrive (senza guardarsi allo specchio che immaginiamo veritiero almeno quanto l'obiettivo di Nadar) come uno scimmione di "abietta lussuria e bassi istinti" (p. 31), perfettamente a suo agio fra i più scomposti e volgari lazzaroni (cfr. p. 69). Se la litania di insulti fosse limitata a re Ferdinando la cosa si potrebbe pure accettare vista la parte che gli spetta nella

18 Sarà stato, come dice Croce, un atto di obbedienza di un soldato all'ordine del governo inglese. Ma, allo stesso modo, non può, o meglio, non poteva, esserci ammirazione e orgoglio per Nelson da parte dei napoletani. C'è anche da ricordare che su suo ordine era stata distrutta buona parte della flotta napoletana affinché non cadesse in mano francese. 
giusta glorificazione della rivoluzione partenopea (rivoluzione di Championnet per Dumas), ma lo scrittore va ben oltre nel dimostrare il suo amore per Napoli e la cosa non poteva passare inosservata per i lettori dell'"Indipendente":

Ogni popolo ha avuto il suo re che ha impersonato lo spirito della nazione: gli scozzesi Robert Bruce, gli inglesi Enrico VIII, i tedeschi Massimiliano, i russi Ivan il Terribile, i polacchi Giovanni Sobieski, gli spagnoli Carlo V, i francesi Enrico IV. I napoletani hanno avuto Nasone. (p. 192)

Poco più avanti Dumas rincara la dose. E questa volta non c'entra proprio re Ferdinando, si parla solo del popolo napoletano: "Un popolo instabile, superstizioso, ignorante, feroce" (p. 197). $\mathrm{E}$ ancora, ma questa è calunnia vera e propria, mille volte ribaltata nelle pagine di Mastriani: "Una delle caratteristiche della popolazione napoletana è la sua ripugnanza istintiva a recare aiuto a un suo simile, anche se in pericolo di morte" (p. 236).

Si potrebbe continuare con altri grotteschi esempi di retorica dumasiana. C'è perfino un'accusa di facili costumi e bassa moralità a Napoli a differenza delle "nostre capitali del Nord" (p. 172). Evidentemente Dumas non aveva letto I vermi con le sue statistiche e percentuali sulla prostituzione nelle capitali europee. "Roba da pazzi", avranno pensato i napoletani gratificati da tanto amore "appassionato e fervido". E questo è il Dumas messaggero di civiltà, favorito da Garibaldi aspirante scrittore che gli apre l'archivio segreto dei Borboni, nominato direttore del museo di Napoli e degli scavi di Pompei, ben alloggiato al casino reale di Chiatamone da dove emana i suoi oracoli di verità, saggezza e libertà. Chissà perché i napoletani, e Mastriani fra loro, non lo capirono.

Claude Schopp, curatore della più recente edizione de La San Felice (Parigi Gallimard, 1996), dice che il romanzo di Dumas "è un canto alla gloria dei repubblicani francesi scritto sotto il Secondo Impero" (MARCHI, 1999, p. 1753). Certo, il romanzo può anche essere questo, ma visto da Napoli vi si scorge una mal celata stizza e fors'anche un'infantile vendetta di vecchio rancoroso, di parolaio ambizioso e insoddisfatto. Sì perché i suoi insulti alla cultura di Napoli vengono quando ha già deciso di lasciare la città che non lo vuole e quando è già rientrato Parigi.

I tre anni passati a Napoli da Dumas portano dunque, nelle parole del suo critico, a "un canto alla gloria dei repubblicani francesi” (MARCHI, 1999, p. 1753)? E c'era bisogno di passare tre anni a Napoli per cantare questa canzone? Portano invece, ci pare con obiettività, 
al riconoscimento di un fallimento, a un'esperienza da dimenticare se ci sono voluti 192 interminabili capitoli per una causa così scontata, anacronistica e irrilevante negli anni Sessanta. Neanche ai napoletani piaceva il Secondo Impero, bien entendu, e Mastriani, in particolare, aveva per esso un sentimento misto di rabbia e disprezzo. Ma nessuna simpatia suscitava Dumas con l'esaltazione della Prima Repubblica (se pur è giusta l'interpretazione di Schopp) parallela a quella Partenopea.

Pare ovvio, a questo punto, che bisognava in qualche modo rispondere alla storia napoletana del francese, se non altro per una questione di orgoglio. E Mastriani sentì il bisogno - senz'altro per sollecitazioni esterne, come lui stesso dichiara - di rispondere per le rime, ovvero a romanzo storico con romanzo storico, stesso titolo e stesso tema. Il titolo non rispecchia l'amplissimo tema di Dumas né per ovvia, ragionata scelta, lo rispecchia in Mastriani. Il quale, cambiandolo nella seconda edizione, dimostra appunto che la prima era una risposta a Dumas.

Mastriani non voleva comunque polemizzare apertamente, aprire una disputa letteraria, o storica addirittura con chi aveva avuto le chiavi dell' archivio segreto borbonico e se ne era vantato. Sarebbe stata cosa aliena alla sua personalità (peraltro sempre generosa nel giudizio di altri scrittori) e comunque assolutamente impensabile per la disparità delle forze, anche mediatiche (“'L'Indipendente' restò attivo per vari anni dopo la partenza del suo fondatore”), e per gli appoggi influenti. Per Mastriani si trattava piuttosto di emendare pacatamente i giudizi negativi del francese sul popolo napoletano, Ferdinando e lazzaroni compresi. Se c'è un punto nella San Felice di Dumas che deve aver irritato Mastriani al massimo grado e sul quale egli sentì il dovere di rettificare è una specie di maledizione che il francese lancia contro i 'borboniani' di tutti i tempi: "Sappiamo che furono dei ben miserabili alleati coloro che, in ogni epoca, difesero la causa dei Borboni” (p. 1154). Per Mastriani, apprezzato scrittore nella Napoli borbonica, che per le nozze di Francesco II aveva glorificato la memoria di Carlo III esaltando il valore autoctono della cultura napoletana, delle leggi fatte a Napoli per i napoletani, questo era veramente troppo, era addirittura un'offesa personale.

Luigia Sanfelice è forse il più storico fra i "romanzi storici”, così descritti nel frontespizio, di Francesco Mastriani. Non solo perché la vicenda della "madre della patria", come fu chiamata la protagonista, è intessuta ai fatti della Repubblica Partenopea, che "gittò il primo germe dell'unità 
italiana"(CROCE, 1998, p. 12), ma anche perché in questo romanzo Mastriani conferma, consolida e definisce il suo ruolo di scrittore come figura pubblica con una chiara funzione sociale, una funzione eminentemente educativa. $\grave{E}$ in questa luce che va vista la sua scrittura di un romanzo-rettifica, con lo stesso titolo (filologicamente migliorato) del monumentale romanzo del famosissimo Alexandre Dumas. Lo scrittore francese, amico di Garibaldi ma mal sopportato dai napoletani, aveva lasciato la città e il bell'alloggio al casino reale di Chiatamone senza un briciolo della gloria che da loro s'aspettava, e anzi con un grosso carico di rancori che affiorano senza troppo garbo ne La San Felice. Mastriani scrivendo Luigia Sanfelice non cerca la sfida, ché sarebbe stata cosa ingenua e velleitaria, ma una rettifica, appunto, ovvero un chiarimento del punto di vista napoletano nei fatti della rivoluzione del Novantanove, soprattutto in rapporto al carattere dei suoi protagonisti, da re Ferdinando all'ultimo dei lazzari di Santa Lucia. Luigia Sanfelice è il più storico dei romanzi di Mastriani, infine, perché con esso si precisa uno stile, un metodo retorico di raccontare i fatti e di rifletterci sopra. La form of fiction predominante è l'anatomia, cui si associa quella del novel, del romanzo vero e proprio, il che vuol dire che, stilisticamente parlando, Luigia Sanfelice corrisponde perfettamente ai contemporanei Misteri di Napoli. ${ }^{19}$

Come indicato in più occasioni, ovvero in più commenti a diversi romanzi, Mastriani preferisce la descrizione alla narrazione. Non che i due orientamenti di scrittura non possano esistere in maniera bilanciata, ma è un fatto che la preferenza accordata alla descrizione comporta un particolare rilievo assegnato allo spazio (agli spazi urbani descritti dettagliatamente, per esempio) e un certo qual disdegno per il racconto lineare, cronologicamente organizzato, cioè per la narrazione pura e semplice. Ho già detto altrove delle implicazione di questi due diversi orientamenti di scrittura in ambito propriamente retorico, e quindi delle forms of fiction di volta in volta adottate e ideate da Mastriani. ${ }^{20}$

In Luigia Sanfelice ci troviamo subito di fronte a un paio di peculiarità nella distribuzione del materiale narrativo. La descrizione iniziale dei personaggi e delle situazioni nella prima parte (delle tre, a numerazione indipendente, di cui è composto il romanzo) è circoscritta nei vari capitoli che si presentano come entità quasi del tutto indipendenti ${ }^{21} \mathrm{e}$ che fanno riferimento a episodi che si incontreranno soltanto molto più avanti nelle pagine del romanzo. Questo ci ricorda che siamo di fronte a una dispositio 'a mosaico', comune nell'anatomy, piuttosto che

19 Per anatomia, novel e altre forms of fiction rimando al mio "Le forme del romanzo di Francesco Mastriani". 20 Faccio riferimento ancora una volta al mio "Le forme del romanzo etc.".

21 Esemplare è il caso del capitolo IX della prima parte. È la storia di "Tommaso il guardiano", che entra nel racconto soltanto perché "venuto in Napoli da pochi anni colla moglie e una figliuola bellissima, fu raccomandato [...] dal cardinale Fabrizio Ruffo, suo compaesano" (I.90) e ammesso come guardaboschi a San Leucio. Ferdinando seduce la figlia di Tommaso e questi gli spara una schioppettata, ma manca il bersaglio: "Fischia la palla agli orecchi del re e gitta in frantumi uno specchio ch'era sull'opposta parete. [...] Da che dipendono i destini de' popoli! Se la palla avesse toccato la tempia del re, Napoli non avrebbe forse veduto le stragi del 99 e gli innumerevoli afforcamenti che seguirono" (I.100). Ferdinando reagisce aizzandogli contro Mercurio, "un cane di quella razza terribile di Terranova che fa spavento anche ai leoni" (I.99) e "poco appresso, alla breve distanza distanza d'un tiro di schioppo, furono udite grida strazianti” (I.100). Fine del capitolo: di Luigia Sanfelice nemmeno l'ombra. 
di fronte a una narrazione lineare, cronologica dei fatti. E poi dobbiamo rilevare la quasi totale assenza della "protagonista", ovvero del personaggio di Luisa Sanfelice, nella prima parte, che è poi la più lunga delle tre. Dei fatti che hanno reso famosa la nobildonna napoletana si parla, succintamente, soltanto nella seconda parte e nella conclusione della terza. Il titolo del romanzo, dunque, sembra quanto meno inadatto; Mastriani ovviamente l'ha mantenuto come preciso richiamo all'opera di Dumas. Il resto del romanzo, ovvero la parte quantitativamente maggiore di esso, è tutto "contesto", al punto che un titolo diverso, più comprensivo, del tipo "La Rivoluzione Partenopea del 1799" sarebbe stato certamente più accurato.

Le scelte retoriche di Mastriani si spiegano sì con la sua propensione per la descrizione (e, va aggiunto, per le digressioni che comunque sono più che contenute rispetto a quelle chilometriche dello scrittore francese), ma si spiegano anche con il fatto che la storia della Sanfelice secondo la narrazione di Dumas era di dominio pubblico, il che costituiva un punto fermo, un dato acquisito per i lettori, che occorreva tener presente. Dice infatti Mastriani stesso nell'avviso in testa al volume: “[...] Stimai attenermi alla dipintura degli attori principali di quel lugubre dramma; perocché già la Sanfelice era rinata sotto la fervida e immaginosa penna d'uno de' più popolari romanzieri francesi, Alessandro Dumas padre."

La "immaginosa penna" è rispettata da Mastriani che sicuramente conosce la vera storia della Sanfelice, nata Molina, madre di tre figli e con un matrimonio traballante: una figura sicuramente meno eroica di quella leggendaria descritta da Dumas. ${ }^{22}$ Se al centro dell'attenzione del narratore nel romanzo francese non c'è il personaggio della Sanfelice (e La San Felice risulta un titolo che non corrisponde al testo) è perché il romanzo stesso crebbe in tipografia, nel senso che non era certamente compiuto, neanche nella mente dello scrittore, quando con quel titolo se ne cominciò a Parigi nel 1863 la pubblicazione a puntate su "La Presse". La scelta del titolo di Mastriani, che cercava il confronto, era invece obbligata. Esaurita la sua funzione di richiamo polemico, il titolo si poteva anche cambiare e infatti nel 1876 il romanzo riappare come Due feste al mercato. Anche in Mastriani non è centrale la figura della Sanfelice e vistoso rilievo assumono, nel racconto, altri personaggi; primo, fra questi, quello di Eleonora Pimentel Fonseca, di cui Mastriani descrive con ammirazione le doti intellettuali, morali, e il patriottismo appassionato. ${ }^{23} \mathrm{E}$, naturalmente, si scende nei dettagli della descrizione, non solo fisica, della coppia reale, Ferdinando e Carolina, e dei loro amori e delle loro turpitudini

Con tono un po' risentito lo scrittore osserva in apertura che il libro non ebbe molto successo

22 Maggiori dettagli biografici della Sanfelice si trovano in Croce, "Luigia Sanfelice e la congiura dei Baccher", capitolo del citato La rivoluzione napoletana del 1799.

23 Le Due feste al mercato del secondo titolo del romanzo alludono alle esecuzioni capitali, in Piazza del Mercato, della Sanfelice e della Pimentel Fonseca. Il testo del romanzo del 1876 è identico a quello del 1870. Nell' "Avvertenza" delle Due feste al mercato si legge: "Questo mio storico lavoro fu pubblicato in Napoli per la prima volta cinque anni or sono, con altro titolo che poco rispondeva al concetto dell'opera". 
"avvegnaché importante ne fusse il subbietto". Il successo sperato, possiamo ipotizzare, poteva essere lo scuotere la coscienza dei napoletani sulla propria identità storica. E ora che non c'è più il confronto con il romanzo francese, l'opera si può associare ad altre in cui lo stesso argomento storico è stato "a lungo" narrato, ai Lazzari e ai Misteri di Napoli.

Delle tre parti del romanzo di Mastriani, la prima parte è occupata, per un buon tratto, dalla figura sinistra del Cardinal Fabrizio Ruffo. Mastriani ne tratteggia una biografia, che è poi un elenco di nefandezze, conclusa con la 'crociata' del Sanfedismo. Prima e dopo di questa fase cruciale della biografia del porporato, Mastriani presenta il Ruffo dialogante con Eleonora Pimentel Fonseca. Le chiede amore, prima in cambio del salvataggio politico, e poi in cambio della vita. In entrambi i casi la donna dà la misura dell'eroismo che la porterà di lì a poco al patibolo. La seconda parte è dedicata alla breve vita della Repubblica Partenopea e alla fallita congiura dei Backer ${ }^{24}$ che diede alla Sanfelice, per averla rivelata, prima il titolo di "madre della patria" e poi la morte in Piazza del Mercato. La terza parte contiene il resoconto del calvario della Sanfelice per evitare il patibolo (la falsa gravidanza sostenuta da medici napoletani di orientamento liberale) e la macabra fine della nobildonna.

Una miriade di personaggi spunta dalle numerose trame del racconto. Al comparire della Pimentel Fonseca, per esempio, dopo il profilo biografico che già offre occasione per ulteriori digressioni, Mastriani non può fare a meno di dare una rassegna delle presenze più illustri del suo cenacolo. E così incontriamo il grande medico Domenico Cirillo, Gaetano Filangieri (morto prima della rivoluzione), Mario Pagano e, naturalmente, fra loro la Sanfelice. La quale è a Napoli da tutti ammirata, in particolare da due aitanti giovani: dall'uomo cui ha giurato eterna fedeltà, Leonida Ferri (rientrato a Napoli per far la sua parte nella rivoluzione), e dal reazionario borbonico Gerardo Backer impazzito addirittura d'amore per lei. Il Backer innamorato le rivelerà, per salvarle la vita, che una congiura porterà pochi giorni dopo alla fine della Repubblica. A questa prova d'amore del Backer per Luigia corrisponde quella di lei per Leonida Ferri. La Sanfelice offre a lui, all'uomo che ama, il salvacondotto datole dal Backer. Leonida Ferri svela la congiura ai compagni repubblicani. La congiura viene bloccata e il Backer ucciso. Poco dopo anche Leonida Ferri viene ucciso.

La solitudine della Sanfelice nel corso della reazione borbonica ne acuisce il dramma. Sarà lei l'ultima rappresentante della Repubblica ad essere giustiziata. Sarà uccisa da un boia improvvisato, "mal pratico", che fece scempio del suo corpo prima di decapitarlo con un coltellaccio da beccaio: dopo averle staccato per sbaglio una spalla con la mannaia, al

24 "Baccher", in Croce. "Backer" è la forma usata da Mastriani. 
grido di orrore del popolo, il macellaio chiamato a sostituire il boia mastro occupato altrove, ricorse allo strumento a lui più familiare per finire il lavoro. Non mancano nel romanzo altre immagini macabre come questa. Ricordiamo che cronologicamente siamo molto vicini ai Misteri e quindi ritroviamo nella Sanfelice molti aspetti delle tematiche lì ampiamente rappresentate. Retoricamente parlando, è comunque Luigia Sanfelice un romanzo propriamente storico e quindi i temi gotici, frenologici, fisiognomici e perfino morali (pensando alle non infrequenti tirate moraleggianti in calce a descrizioni di fatti e persone) passano in secondo piano.

Emergono invece brillanti i ritratti di Ferdinando e Maria Carolina, di Monsú Attone (Sir John Acton), di William Hamilton e della giovane moglie Emma, amante di Horatio Nelson e della regina. Su tutti spicca per tratti di nobiltà la figura dell'ammiraglio Francesco Caracciolo, fatto ignominiosamente impiccare da Nelson, il quale sarà anche un eroe per gli inglesi e per Dumas, ma certamente non per Mastriani. ${ }^{25}$

C'è poi un lungo catalogo di eroi noti e poco noti della Repubblica Partenopea, dal generale Gabriele Manthoné a Luigi Serlio (un cieco che volle partecipare alla battaglia per la difesa della città dall'orda della Santa Fede). Un'intera rassegna viene poi fuori dalle figure delinquenziali associate ai Borboni: lazzari, briganti, Fra Diavolo, Mammone, il famoso boia Masto Donato... fino al crudelissimo menino di Ferdinando, Gennaro Rivelli. Tutto questo comunque non deve indurci a credere che ci siano soltanto personaggi a tutto tondo, soltanto positivi o soltanto negativi nel romanzo. Anzi, il pensiero di Mastriani raggiunge punte di ispirata penetrazione psicologica quando di un personaggio del tutto perverso, com'è il Ruffo per esempio, nota notevoli doti d'umanità e di senso di giustizia. Lo stesso re Ferdinando, uomo volgare, pusillanime, di squallidi costumi e di vertiginosa ignoranza, mostra a tratti lampi di intelligenza e di arguzia, e addirittura di simpatia, soprattutto in rapporto alla irredimibile abiezione della scellerata sua consorte Carolina d'Austria. "Il caso di ambiguità più notevole è forse quello che riguarda i lazzari che saranno pur ignoranti e violenti, ma allo stesso tempo sono ottimi rappresentanti del generoso e indomabile popolo partenopeo. Se è vero che si battono contro Championnet, e quindi contro gli ideali patriottici e liberali, è pur vero che si battono da eroi per la difesa della patria. Torna qui il senso della "indipendenza nazionale" che aveva fatto grande Carlo III nell'Omaggio sebezio. ${ }^{26}$ Il brano che segue va letto in risposta alle fiorite pagine del

25 È possibile che sia la barbara impiccagione del Caracciolo, sia la distruzione delle navi napoletane dopo la fuga a Palermo di Ferdinando e Carolina, 'imprese' che portano il suggello dell'ammiraglio inglese e costituiscono le macchie della sua condotta altrimenti eroica, non siano altro che atti di obbedienza ad ordini segreti del governo inglese (Cfr. Croce, 1998, p. 16-18). A questa 'giustificazione' forse anche Mastriani, senza ricerche d'archivio, era arrivato. Rimane per lui il fatto che l'uccisione del Caracciolo fu un atto di infamante, irredimibile abiezione.

26 Omaggio sebezio è il titolo della pubblicazione celebrativa per le nozze del principe ereditario del Regno delle Due Sicilie Francesco d'Assisi Maria Leopoldo di Borbone e della principessa Maria Sofia Amalia di Baviera cui parteciparono 40 "penniferi" - così chiamava gli scrittori re Ferdinando II, padre dello sposo - fra cui Francesco Mastriani nel cui contributo, "Ricordi storici sulla fondazione della Dinastia Borbonica nel Reame delle Due Sicilie", era esaltata la magnanimità di Carlo III, fondatore della dinastia. 
Dumas sulla 'conquista' di Napoli da parte di Championnet.

Championnet moveva intanto da Roma per Napoli con venticinquemila uomini, con artiglierie, viveri e macchine guerresche. Quattro divisioni componevano il suo esercito. Non lieve resistenza ebbe a superare il generale francese non tanto dalle poche forze di terra e di mare che accanitamente difesero i regi diritti, quanto dagli informi eserciti di Fra Diavolo, Pronio, Salomone, Rodio e Mammone, pe' quali grande sgomento entrò nell'esercito francese. Ma dove si compirono fatti straordinari ed incredibili si fu alle porte stesse della città di Napoli per l'audace resistenza che i francesi ebbero a sperimentare da' nostri lazzari. È questa sì certo una delle pagine più gloriose della storia di Napoli. Noi non guardiamo nella eroica difesa de' lazzari napolitani che il sentimento della indipendenza nazionale e l'odio allo straniero. Che se in questo nobile sentimento andava pur confusa la causa della tirannide, non è per ciò da estimarsi men glorioso il fatto e meno eroica la difesa. Qualunque sia la causa che se ne giovi, la difesa delle patrie zolle contro l'invasione straniera è santa, è legittima. Essa sola giustifica e assolve quella mostruosa infamia che è la guerra. Così il popolo non avesse trovato spesso nella corruzione delle classi elevate una viva opposizione allo sviluppo del sentimento della indipendenza nazionale! Napoli non avrebbe avuto a gemere per lo spazio di oltre sette secoli sotto barbare straniere signorie. (2.5-6)

Il genio militare dello Championnet, che tanto risplendeva in Dumas contro l'incapace generale Mack a Velletri e a Civita Castellana, non sembra tanto ovvio negli scontri con i briganti in Terra di Lavoro e, soprattutto, con in lazzari alle porte di Napoli. Quel che è evidente, invece, è l'orgoglio napoletano del narratore che rettifica la prospettiva degli eventi storici. I fatti sono gli stessi raccontati da Dumas, ma 'la storia' è molto diversa.

Luigia Sanfelice, infine, è certamente sia romanzo che storia ed illustra bene lo stile, ovvero la forma ibridata di novel e anatomy in cui eccelle Mastriani. Per quel che riguarda la veridicità dei fatti narrati (un elemento da valutare rispetto alla vocazione didattica del romanziere) bisogna dire che le licenze poetiche riguardano soprattutto le vicende della Sanfelice e arrivano a Mastriani da Dumas. Non corrispondono certamente al vero. Luigia Sanfelice, nata Molina, all'epoca dei fatti narrati era tutt'altra cosa dalla "protagonista" del romanzo. Era una donna di 
mondo, con diversi amanti, un marito, tre figli e molti debiti. Mastriani non tradisce la verità, accetta la leggenda. Non è su questo, come abbiamo visto, che sceglie di confrontarsi con Dumas. In apertura d'opera, aveva del resto dichiarato di non aver intenzione di modificare quella che ormai era divenuta la vulgata, ovvero la leggenda della Sanfelice.

\section{Riferimenti Bibliografici}

ADDESSO, Cristiana Anna; MASTRIANI, Emilio; MASTRIANI, Rosario. Che somma sventura è nascere a Napoli! Bio-bibliografia di Francesco Mastriani. Roma: Aracne, 2012.

BRESCIANI, Antonio. L'ebreo di Verona. Torino: Tipografia Eredi Botta, 1850.

COLLETTA, Pietro. Storia del Reame di Napoli dal 1734 sino al 1825. Firenze: Le Monnier, 1856.

CROCE, Benedetto. La rivoluzione napoletana del 1799. Napoli: Bibliopolis, 1998.

DI FILIPPO, Antonio. Lo scacco e la ragione: gruppi intellettuali, giornali e romanzi nella Napoli dell'800: Mastriani. Lecce: Milella, 1987.

DUMAS, Alexandre (padre). La Sanfelice. Trad. Fabrizio Ascari, Graziella Cillario e Piero Ferrero. Milano: Adelphi, 1999. 2 v.

. La San Felice. Ed. Claude Schopp. Paris: Gallimard, 1996.

GUARDIANI, Francesco. Le forme del romanzo di Francesco Mastriani. Critica letteraria 134.1, 25, p. 95-113, 2007.

MARCHI, Ena. A Napoli, nel nome del padre e della Rivoluzione. In: DUMAS, Alexandre (padre): La Sanfelice. Trad. Fabrizio Ascari, Graziella Cillario e Piero Ferrero. Milano: Adelphi, 1999. p. 1743-1754. $2 \mathrm{v}$.

MASTRIANI, Filippo. Cenni sulla vita e sugli scritti di Francesco Mastriani. In: MASTRIANI, Francesco. Le ombre. A cura di Luca Torre. Napoli: Torre Editrice, 1992. v. 2, p. 725-884. I Cenni sono stati recentemente ristampati in: ADDESSO, Cristiana Anna; MASTRIANI, Emilio; MASTRIANI, Rosario. Che somma sventura è nascere a Napoli! Bio-bibliografia di Francesco Mastriani. Roma: Aracne, 2012. p. 127-279.

MASTRIANI, Francesco. Ricordi storici sulla fondazione della Dinastia Borbonica nel Reame delle Due Sicilie. In: Omaggio sebezio. Napoli, s.a., ma 1859. . Acaja. Napoli: Gargiulo, 1860. . Angiolina. Napoli: Tipografia dell'Industria: v. 1-2, 1858; v. 3, 1859. 3 v.

Cenere. Napoli: Edizioni Ginka, 1975. (Prima edizione: Appendice del "Roma", 28 maggio-14 agosto, 1877). . Eufemia, ovvero Il segreto di due amanti. Napoli: Gargiulo, 1868. 
Due feste al mercato. Napoli: Gabriele Regina, 1876.

Karì-Tismé. Memorie d'una schiava. Appendice del Roma, 15 dicembre 1882 - 24 febbraio1883. . I figli del lusso. Séguito dell'opera I vermi. Napoli: Gargiulo, 1866. 4 v.

. I lazzari. Romanzo storico Napoli: Attività Bibliografica Editoriale, 1976. (Prima edizione:

Napoli: Gargiulo, 1865).

. I misteri di Napoli. Studi storico-sociali. Napoli: Stabilimento Tipografico del Commend. G. Nobile, 1869-1870. 2 v.

I vermi tudi storici su le classi pericolose in Napoli. Napoli: Gabriele Regina, 1877-78. (Prima edizione: 10 v. Napoli: Gargiulo, 1863-64).

. Il campanello dei Luizzi: cronaca napoletana del 1799. Appendice del "Roma", 10 aprile -6 agosto, 1885 .

. Il figlio del diavolo. Napoli: Francesco Casella fu Gennaro, s.a. 4 v. Prima edizione: Napoli: Achille Di Pierno, 1869. 3 v.

.Il padrone della vetraia all'Arenaccia. Appendice del "Roma", 19 giugno-11 luglio, 1890.

. La brutta. Napoli: Gabriele Regina, 1879. 3 v. Prima edizione: In appendice de "La Domenica" I.2 (18 nov. 1866) - II.48 (6 ott. 1867).

. La contessa di Montès. Milano: Barion, 1925. Prima edizione: 3 v. Napoli: Gargiulo 1873.

Antonio Di Filippo indica l'anno 1869 per la prima edizione, senza indicazione di luogo o stampatore.

. La figlia del croato. Romanzo storico. Napoli: Giosuè Rondinella, 1877. Prima edizione: In appendice de "La Domenica" II.9 (6 gen. 1867) - II.43 (1 sett.1867).

. La Medea di Porta Medina. Firenze: Salani, 1931. Prima edizione: In appendice del "Roma", 12 ottobre-29 dicembre, 1881 .

. Le ombre. Lavoro e miseria. A cura di Luca Torre. Napoli: Torre Editrice, 1992. 2 v. Contiene i Cenni sulla vita e sugli scritti di Francesco Mastriani, di Filippo Mastriani (v. 2, p. 725-884). Prima edizione: Napoli: Gargiulo, 1868.

. Luigia Sanfelice. Romanzo storico. Napoli: Stabilimento Tipografico della Sirena, 1870.

Memorie di una monaca. Napoli: Gabriele Regina, 1879.

. Una martire. Napoli: Salvati, s.a. Prima edizione. Napoli: Gargiulo,1868. 5 v. Antonio Di

Filippo ( $v d$.) indica una prima edizione su una irreperibile terza annata de "La Domenica" del 1868, 17 appendici, dal 9 agosto al 13 dicembre. 